\title{
Surface doping and stabilization of Sil111) with boron
}

\section{Citation}

Bedrossian, P., Robert D. Meade, K. Mortensen, D. M. Chen, J. A. Golovchenko, and David Vanderbilt. 1989. Surface Doping and Stabilization of Sil111) with Boron. Physical Review Letters 63, no. 12: 1257-1260. doi:10.1103/physrevlett.63.1257.

\section{Published Version}

doi:10.1103/PhysRevLett.63.1257

\section{Permanent link}

http://nrs.harvard.edu/urn-3:HUL.InstRepos:29407036

\section{Terms of Use}

This article was downloaded from Harvard University's DASH repository, and is made available under the terms and conditions applicable to Other Posted Material, as set forth at http:// nrs.harvard.edu/urn-3:HUL.InstRepos:dash.current.terms-of-use\#LAA

\section{Share Your Story}

The Harvard community has made this article openly available.

Please share how this access benefits you. Submit a story.

Accessibility 


\title{
Surface Doping and Stabilization of Si(111) with Boron
}

\author{
P. Bedrossian, ${ }^{(1,2)}$ Robert D. Meade, ${ }^{(1)}$ K. Mortensen, ${ }^{(1,2)}$ D. M. Chen, ${ }^{(1,2,3)}$ J. A. Golovchenko, ${ }^{(1,2)}$ \\ and David Vanderbilt ${ }^{(1)}$ \\ ${ }^{(1)}$ Lyman Laboratory, Harvard University, Cambridge, Massachusetts 02138 \\ ${ }^{(2)}$ Rowland Institute for Science, Cambridge, Massachusetts 02142 \\ ${ }^{(3)}$ Physics Department, University of Western Ontario, London, Ontario, Canada N6A $3 K 7$ \\ (Received 3 April 1989)
}

\begin{abstract}
We have investigated the incorporation of boron into the $\operatorname{Si}(111)(\sqrt{3} \times \sqrt{3}) R 30^{\circ}$ surface from low boron concentration up to $\frac{1}{3}$ monolayer, using tunneling microscopy and spectroscopy and first-principles total-energy calculations. Surprisingly, we find that a $(\sqrt{3} \times \sqrt{3}) R 30^{\circ}$ structure composed almost entirely of $\mathrm{Si}$ adatoms on a $\mathrm{Si}$ double layer can be stabilized by boron doping in near-surface layers. Moreover, when boron atoms are in the surface layers, the adatom site is unfavorable compared with the site underneath the adatom, unlike other group-III elements adsorbed on the $\mathrm{Si}(111)$ surface.
\end{abstract}

PACS numbers: $68.35 . \mathrm{Dv}, 61.16 . \mathrm{Di}, 73.20 . \mathrm{Hb}$

Recent studies have revealed that $\mathrm{Al}, \mathrm{Ga}$, and In all show quite similar behavior when adsorbed on $\mathrm{Si}(111)$, and it might be thought that adsorption of group-III metals on this surface was well characterized and understood. ${ }^{1-5}$ In this Letter, we report two entirely novel features associated with the presence of boron, another group-III element, on the $\mathrm{Si}(111)$ surface. Using scanning tunneling microscopy (STM) and spectroscopy, we find that a large subsurface concentration of boron can stabilize a $(\sqrt{3} \times \sqrt{3}) R 30^{\circ}$ structure of $\mathrm{Si}$ adatoms on a $\mathrm{Si}$ double layer. To our knowledge, such stabilization has not previously been reported, and we propose a doping mechanism to explain it. Second, we determine from first-principles total-energy calculations that when boron is observed at the surface, it prefers the site directly underneath the adatom, in dramatic contrast to other group-III adsorbates, all of which occupy the adatom site. ${ }^{2,5}$ Our results demonstrate the feasibility of modifying the atomic-scale morphology and electronic structure of semiconductor surfaces by heavily doping the near-surface region.

Three techniques were employed to prepare $\mathrm{Si}(111)$ $(\sqrt{3} \times \sqrt{3}) R 30^{\circ}$ surfaces with various boron doping concentrations. First, heavily boron-doped $\mathrm{Si}(111)$ wafers $\left(\approx 10^{20} \mathrm{~cm}^{-3}\right.$ ) were annealed at $1000^{\circ} \mathrm{C}$ in an ultrahigh-vacuum chamber with a base pressure of $1 \times 10^{-10}$ Torr. This preparation gave rise to a $(\sqrt{3} \times \sqrt{3}) R 30^{\circ}$ reconstruction as characterized by low-energy electron diffraction (LEED). The mechanism for the surface boron segregation that induces this surface lattice has been discussed previously. ${ }^{6}$ As displayed in the tunneling micrograph of Fig. 1(a), a mixture of atomic species is evident. Brighter adatoms are randomly distributed and account for $20 \%-25 \%$ of the atoms imaged.

In the second preparation, boron was sputtered by a 1.0-keV neon-ion beam onto a cleaned $\mathrm{Si}(111) 7 \times 7$ surface (As doped to $0.02 \Omega \mathrm{cm}$ ). Subsequent heating of this surface to $800^{\circ} \mathrm{C}$ yielded the $(\sqrt{3} \times \sqrt{3}) R 30^{\circ}$ LEED pattern. STM investigations revealed that, while short
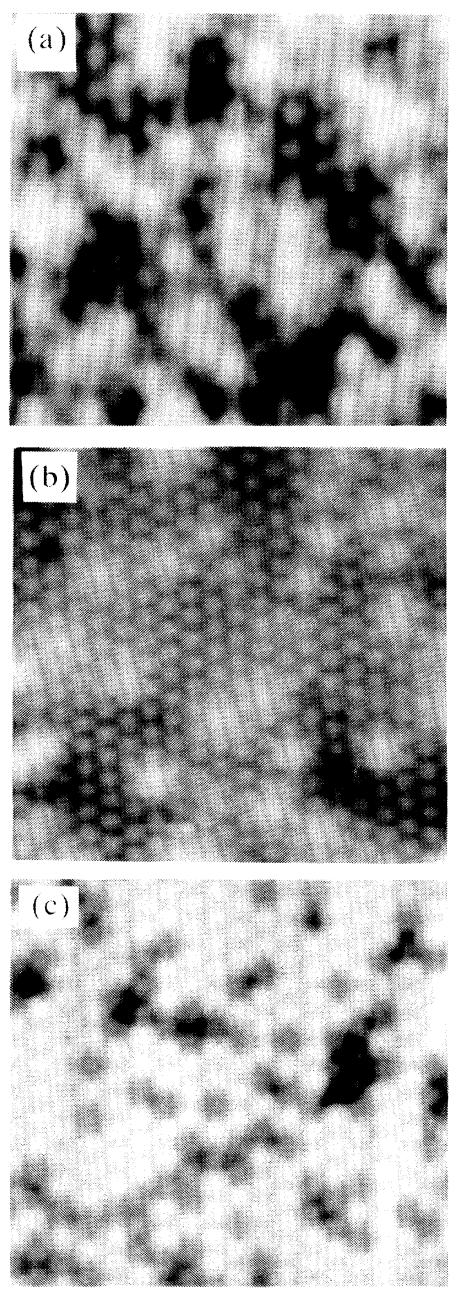

FIG. 1. (a) $100 \times 80-\AA^{2}$ tunneling image showing the $(\sqrt{3}$ $\times \sqrt{3}) R 30^{\circ}$ structure after annealing degenerately borondoped $\mathrm{Si}(111)$ at $1000^{\circ} \mathrm{C}$. Tip bias $=-1.4 \mathrm{~V}, 1 \mathrm{nA}$. (b) $100 \times 100-\AA^{2}$ tunneling image of $\mathrm{Si}(111)$ prepared by sputtering of $\mathrm{B}$ on clean $\mathrm{Si}(111) 7 \times 7$. (c) $100 \times 100-\AA^{2}$ tunneling image prepared by $\mathrm{Si}$ reevaporation on $\mathrm{Si}(111)(\sqrt{3} \times \sqrt{3}) R 30^{\circ}-\mathrm{B}$. 
deposition times yielded topographs similar to Fig. 1(a), increased deposition times resulted in a diminished concentration $(<5 \%)$ of the bright sites, as shown in Fig. 1 (b).

To raise the concentration of these adatoms to a majority, the $(\sqrt{3} \times \sqrt{3}) R 30^{\circ}$ reconstruction was first prepared on a boron-doped $\mathrm{Si}(111)$ wafer as described above, and silicon was then deposited by evaporation from a second $\mathrm{Si}$ wafer until the original LEED pattern was obliterated. Annealing at $800^{\circ} \mathrm{C}$ yielded a $7 \times 7$ diffraction pattern. Further rapid heating $\left(950^{\circ} \mathrm{C}, 20\right.$ sec) reproduced the $(\sqrt{3} \times \sqrt{3}) R 30^{\circ}$ LEED pattern. As shown in Fig. 1(c), the STM revealed that the bright adatoms now account for $80 \%$ of the adatoms.

In general, increased boron concentration at the surface yields more dark adatoms, and increased silicon concentration yields more bright ones. Thus we associate the dark adatoms with the presence of boron, and the bright ones with its absence. This conclusion will be further supported by theory and our electronic measurements. We have also established that all the adatoms in the tunneling images occupy the threefold filled site $\left(T_{4}\right)$ rather than the threefold hollow site $\left(\mathrm{H}_{3}\right)$, by studying the adatom registries on a mixed-phase surface. ${ }^{3,5}$

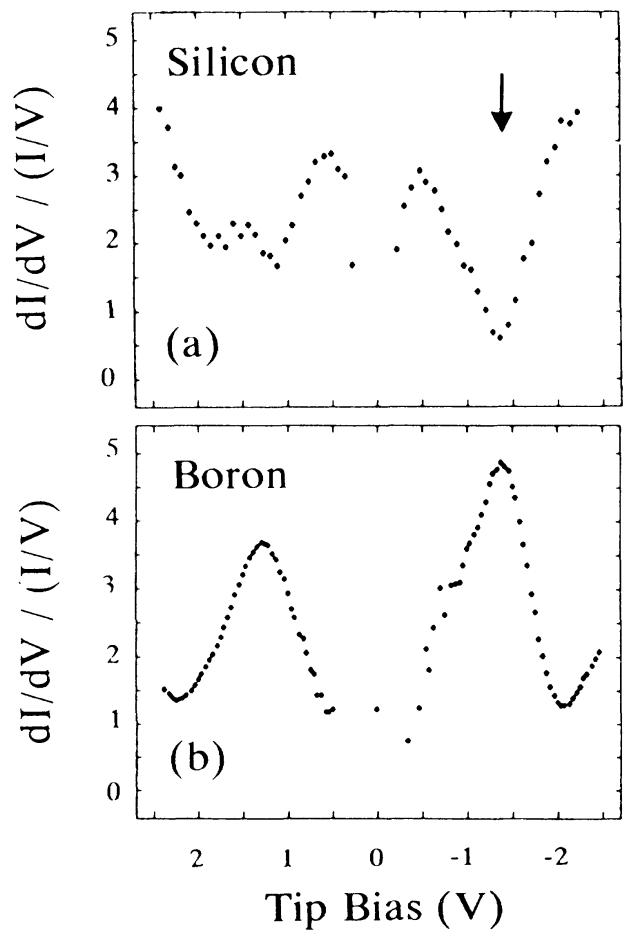

FIG. 2. Normalized differential conductivity spectra recorded on a $\mathrm{Si}(111)(\sqrt{3} \times \sqrt{3}) R 30^{\circ}$-B surface prepared by annealing $\approx 10^{20}-\mathrm{cm}^{3}$-B-doped $\mathrm{Si}(111)$ at $1000^{\circ} \mathrm{C}$ for $2 \mathrm{~min}$. Positive tip bias corresponds to occupied sample states, and the arrow indicates $-1.4 \mathrm{~V}$ tip bias. (a) Spectrum acquired with the tip over a bright atom (Si adatom above silicon). (b) Spectrum acquired with the tip over a dark atom (above boron).
Local tunneling spectra were acquired with the tip directly above the bright or the dark adatoms. As pointed out previously, ${ }^{7}$ the normalized differential conductivity, $(d I / d V) /(I / V)$, reflects the local density of surface states. Typical spectra for each adatom type are shown in Fig. 2. Both display an occupied state at a tip bias of +1.5-1.8 $\mathrm{V}$ regardless of surface boron concentration. Peaks in the tunneling spectra above the bright adatoms also occur within $0.5 \mathrm{eV}$ on either side of the Fermi level. The appearance of this structure for bright adatoms and its absence for the dark atoms accounts for the former's persistent bright appearance in all tunneling images observed regardless of the polarity of the tip bias.

Spectra acquired above dark adatoms, as in Fig. 2(b), display a prominent peak at $-1.4-\mathrm{V}$ tip bias. Since the bright-adatom spectra such as Fig. 2(a) display a minimum at the same bias, indicated by the arrow, the spatial distribution of the unoccupied state atop the dark adatoms is revealed by simultaneously acquiring both a constant-current topograph [Fig. 3(a)] and an image of the differential conductivity [Fig. 3(b)] at $-1.4-\mathrm{V}$ tip bias. It is apparent that peaks in the differential conductivity at this bias occur directly atop dark atoms, while troughs occur directly atop bright ones. We note that the two images are acquired under identical bias and current in order to suppress the contribution of the background in the differential-conductivity image, which results from changes in the transmission probability with
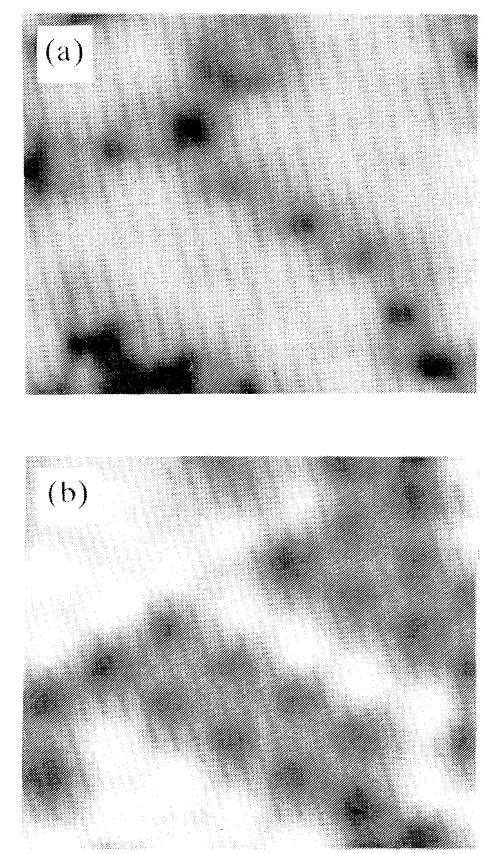

FIG. 3. (a) $100 \times 100-\AA^{2}$ tunneling image recorded at tip bias of $-1.4 \mathrm{~V}, 1 \mathrm{nA}$. (b) Simultaneous image of the differential conductivity at the same bias. Bright regions indicate higher differential conductance. 
the tip height and bias, to the contrast between the two types of atomic sites. ${ }^{8}$

In order to understand the observed structural and electronic features described above, it is important to realize that the actual vertical site of the boron atoms in the $(\sqrt{3} \times \sqrt{3}) R 30^{\circ}$ reconstruction must be established. Two possible positions for the boron atom consistent with our tunneling images are shown in Fig. 4. In case (a), the boron is in the threefold filled adatom position $\left(T_{4}\right)$, and in case (b), it is directly underneath a silicon adatom. We call the latter site $B_{5}$. Other possibilities are ruled out by the preservation in the tunneling images of both the threefold symmetry of the surface and the reflection symmetry of the $\sqrt{3} \times \sqrt{3}$ unit cell about the short diagonal, regardless of the tip bias. A recent report of symmetry breaking ${ }^{9}$ in this system has not been confirmed in this work. To determine the favored structure, we have performed ab initio total-energy calculations for these two possibilities.

The calculations employ nonlocal pseudopotentials and are performed in the local-density approximation (LDA). We use a slab geometry of five double layers (32 atoms per unit cell) separated by three double layers of vacuum. The Hamiltonian is expanded in a basis of plane waves up to $12 \mathrm{Ry}$ and is diagonalized selfconsistently at four $k$ points in the irreducible Brillouin zone. The atomic coordinates are then relaxed until the energies are converged.

We find that the structure with boron occupying the $B_{5}$ site is favored over that with boron in the $T_{4}$ site by $0.93 \mathrm{eV}$ per adatom cell, or $0.31 \mathrm{eV} /(1 \times 1 \mathrm{cell})$. To emphasize the magnitude of this energy difference, we point out that $a b$ initio calculations have shown that the energy difference between an unreconstructed surface and a $2 \times 2$ adatom-covered surface, which is estimated to be close in energy to the $7 \times 7$ reconstruction, is $0.27 \mathrm{eV} /$ $(1 \times 1$ cell $) .{ }^{10}$ In light of the large energy difference favoring boron in the $B_{5}$ site, we conclude that this is the final structure after the annealing procedures used in the present experiment. Therefore, all observed adatoms

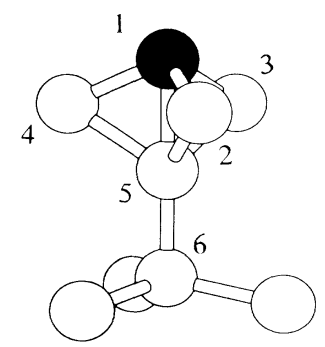

(A)

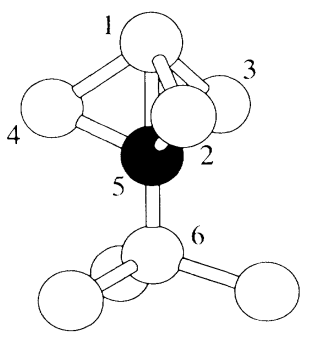

(B)
FIG. 4. Two possible locations of the boron atom: (a) $T_{4}$ adatom, as other group-III adsorbates; (b) $B_{5}$ location, strongly favored. The models are drawn with the relaxed coordinates.
(Fig. 1) are silicon, the dark ones having boron atoms below in the $B_{5}$ site.

Qualitatively, this can be understood as follows. Boron has a smaller covalent radius than silicon, ${ }^{11}$ so the ideal $\mathrm{B}-\mathrm{Si}$ bond length is $12 \%$ shorter than the ideal $\mathrm{Si}-\mathrm{Si}$ bond length. In both configurations considered, the bond-angle stress in the adatom unit cell is reduced by relaxing atoms 2,3 , and 4 inward (see Fig. 4), decreasing their separation by $10 \%$ relative to the bulk atomic separation. In the structure of Fig. 4(a), this induces an unfavorable compression of all bonds to atom 5 , while for structure (b), this inward relaxation is favorable, since atom 5 (the boron atom in this case) prefers smaller bond lengths. By contrast, the other group-III elements have larger covalent radii than $\mathrm{Si}$ and therefore prefer the $T_{4}$ site. In fact, our total-energy calculations predict that for the gallium-covered $\operatorname{Si}(111)(\sqrt{3} \times \sqrt{3})$ $R 30^{\circ}$ surface, the $T_{4}$ site is favored over the $B_{5}$ site by $0.22 \mathrm{eV} /(1 \times 1$ cell $)$. Note that the local stress has been almost entirely relieved by the $B$ substitution on site 5 [Fig. 4(b)], so that there is no driving force for further subsurface $B$ substitution.

A calculated electronic consequence of this model is that charge is transferred to the boron atom from the dangling-bond orbital of the silicon adatom above it, leaving the latter state empty. In particular, our LDA calculations place the unoccupied surface band on the top silicon atoms $0.3 \mathrm{eV}$ higher than the corresponding half-occupied band of silicon adatoms on a silicon substrate. This is in qualitative agreement with the local tunneling spectra, such as Fig. 2(b), although the actual shift relative to the unoccupied state in Fig. 2(a) is somewhat larger. ${ }^{12}$

While a spectrum from boron-free sites [Fig. 2(a)] shows no states at this energy, it does indicate significant density of states within $0.5 \mathrm{eV}$ on either side of the Fermi level, as expected for a silicon adatom on a silicon double layer in a $\sqrt{3} \times \sqrt{3}$ geometry. ${ }^{13}$ This further supports our assignment of such a configuration to the bright adatoms. Though the precise shape of this structure in tunneling spectra is not predicted by LDA calculations, we note that the very appearance of a large density of states surrounding the Fermi level and the minimum underneath the arrow also represent sharp deviations from the reported spectra for other group-III-doped Si(111) surfaces. ${ }^{4}$ The final prominent feature in both Figs. 2(a) and 2(b), the state $1.5 \mathrm{eV}$ below the Fermi level, has been associated with the occupation of backbonding orbitals ${ }^{13}$ and is therefore expected to appear on both boron-occupied sites and boron-free sites.

It is surprising that a $(\sqrt{3} \times \sqrt{3}) R 30^{\circ}$ reconstruction of silicon adatoms on a silicon double layer is stabilized with respect to the familiar $7 \times 7$ surface by strong subsurface boron doping [Fig. 1(c)]. The adatom structures which are observed on clean $\mathrm{Si}(111) 7 \times 7$ and $\mathrm{Ge}(111) c 2$ $\times 8$ surfaces with a lower density than on $\sqrt{3} \times \sqrt{3}$, and 
are therefore accompanied by rest atoms. Such structures involving rest atoms are stabilized by charge transfer from the adatom band to the more favorable rest-atom band. A $\sqrt{3} \times \sqrt{3}$ adatom-covered surface has no rest atoms, and is therefore unfavorable relative to surfaces of lower adatom density despite the fact it has fewer dangling bonds. However, on a doped surface, the unfavorable adatom band may be partially depopulated by transferring electrons to acceptor states. Hence, we suggest that charge transfer from the surface dangling bonds to the boron atoms accumulated in the subsurface layers accounts for the energetic stabilization of the $(\sqrt{3} \times \sqrt{3}) R 30^{\circ}$ over the $7 \times 7$ dimer-adatom-stackingfault structure. This effect saturates when there is one subsurface $B$ substitution per adatom, and therefore higher subsurface doping levels are probably unfavorable.

In conclusion, we have investigated the incorporation of boron into the $\operatorname{Si}(111)(\sqrt{3} \times \sqrt{3}) R 30^{\circ}$ surface from low boron concentration up to $\frac{1}{3}$ monolayer coverage. The boron and silicon species are distinguished with the tunneling microscope and the electronic structures associated with each are revealed by local tunneling microscopy. Total-energy calculations establish that boron occupation of the adatom site is unfavorable compared with the site underneath the adatom; thus the surface band structure observed differs from that of group-III metals on the $\mathrm{Si}(111)$ surface. We have proposed a mechanism of subsurface doping for the remarkable stabilization of the unusual $\operatorname{Si}(111)(\sqrt{3} \times \sqrt{3}) R 30^{\circ}$ reconstruction.

We are grateful to S. Dodge, W. Hill, and J. Segal for valuable technical assistance. This work was supported by ONR Grant No. N00014-87-K-0511, NSF Grant No. DMR-88-17291, the John von Neumann Center, and the Harvard University Materials Research Laboratory. We also acknowledge assistance from NATO (K.M.), NSF (P.B.), Harvard University (R.D.M.), the Sloan Foundation (D.V.), and the Ontario Center for Material Research (D.M.C.).

${ }^{1}$ J. J. Lander and J. Morrison, Surf. Sci. 2, 553 (1964).

2 J. M. Nicholls, B. Reihl, and J. E. Northrup, Phys. Rev. B 35, 4137 (1987); G. V. Hansson, J. M. Nicholls, P. Mårtensson, and R. I. G. Uhrberg, Surf. Sci. 168, 105 (1986).

3 J. Nogami, Sang-il Park, and C. F. Quate, Phys. Rev. B 36, 6221 (1987); Surf. Sci. 203, L631 (1988).

${ }^{4}$ R. J. Hamers and J. E. Demuth, Phys. Rev. Lett. 60, 2527 (1988).

${ }^{5}$ J. E. Zegenhagen, J. R. Patel, Paul Freeland, D. M. Chen, J. A. Golovchenko, P. Bedrossian, and J. E. Northrup, Phys. Rev. B 39, 1298 (1989).

${ }^{6}$ V. V. Korobtsov, V. G. Lifshits, and A. V. Zotov, Surf. Sci. 195, 466 (1988).

${ }^{7}$ R. M. Feenstra, J. A. Stroscio, and A. P. Fein, Surf. Sci. 181, 295 (1987).

${ }^{8}$ G. Binnig and H. Rohrer, IBM J. Res. Dev. 30, 355 (1986);

J. A. Stroscio, R. M. Feenstra, D. M. Newns, and A. P. Fein, J. Vac. Sci. Technol. A 6, 499 (1988).

${ }^{9}$ F. Thibaudau, Ph. Dumas, Ph. Mathiez, A. Humbert, D. Satti, and F. Salvan, Surf. Sci. 211/212, 148 (1989).

${ }^{10}$ D. Vanderbilt, Phys. Rev. Lett. 59, 1456 (1987); R. D. Meade and D. Vanderbilt, Phys. Rev. B 40, 3905 (1989).

${ }^{11} \mathrm{~L}$. Pauling, The Nature of the Chemical Bond, and the Structure of Molecules and Crystals (Cornell Univ. Press, Ithaca, NY, 1960), 3rd ed., p. 246.

${ }^{12}$ Recent inverse photoemission measurements have revealed unoccupied states at 0.5 and $1.4 \mathrm{eV}$ for a $\operatorname{Si}(111)(\sqrt{3} \times \sqrt{3})$. $R 30^{\circ}$-B surface, in good agreement with our observations; $\mathrm{F}$. Himpsel (private communication).

${ }^{13}$ J. E. Northrup, Phys. Rev. Lett. 57, 154 (1986). 

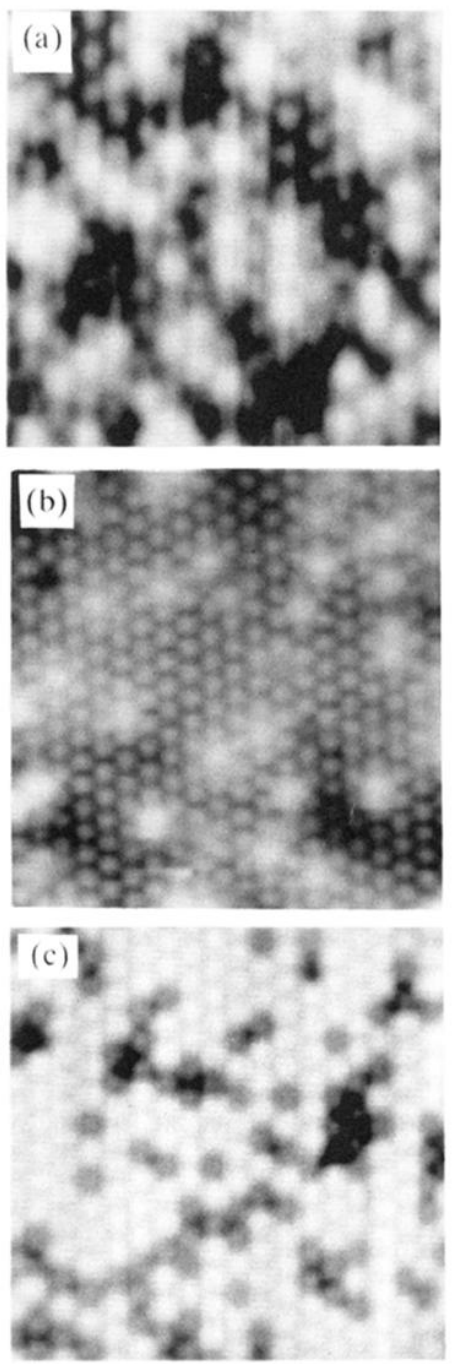

FIG. 1. (a) $100 \times 80-\AA^{2}$ tunneling image showing the $(\sqrt{3}$ $\times \sqrt{3}) R 30^{\circ}$ structure after annealing degenerately borondoped $\mathrm{Si}(111)$ at $1000^{\circ} \mathrm{C}$. Tip bias $=-1.4 \mathrm{~V}, 1 \mathrm{nA}$. (b) $100 \times 100-\AA^{2}$ tunneling image of $\mathrm{Si}(111)$ prepared by sputtering of $\mathrm{B}$ on clean $\mathrm{Si}(111) 7 \times 7$. (c) $100 \times 100-\AA^{2}$ tunneling image prepared by $\mathrm{Si}$ reevaporation on $\mathrm{Si}(111)(\sqrt{3} \times \sqrt{3}) R 30^{\circ}-\mathrm{B}$. 

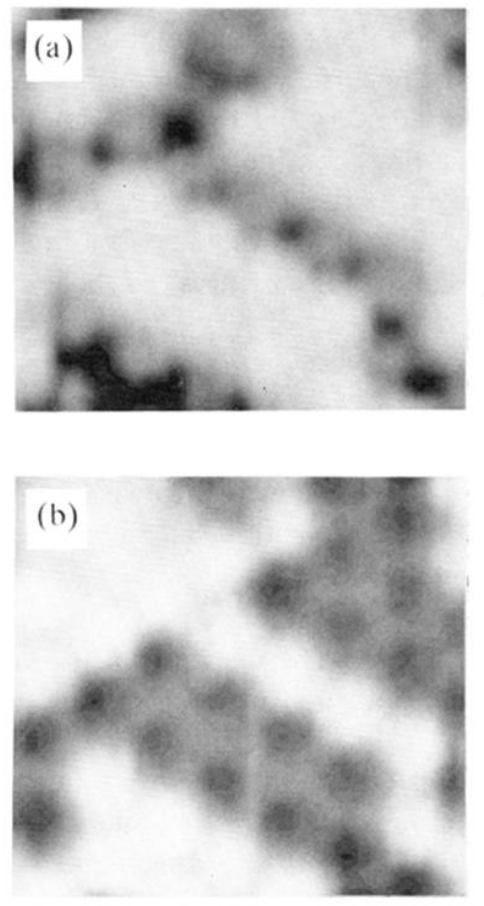

FIG. 3. (a) $100 \times 100-\AA^{2}$ tunneling image recorded at tip bias of $-1.4 \mathrm{~V}, 1 \mathrm{nA}$. (b) Simultaneous image of the differential conductivity at the same bias. Bright regions indicate higher differential conductance. 